\title{
Improvement in Vagal Function in a Post Breast Cancer Patient Receiving Chiropractic Care: A Case Study
}

\author{
Otto J. Janke ${ }^{a, b, ~ e, ~ D a v i d ~ R u s s e l l ~}{ }^{\text {c, d }}$
}

\begin{abstract}
Heart rate variability (HRV) is widely used to demonstrate the vagal function and sympathetic function of the autonomic nervous system and evaluate autonomic dysregulation. Current literature demonstrated that the HRV of women who have gone through breast cancer, surgery and chemotherapy stays low for possibly up to a year or more. The purpose of this paper was to chronicle the consistent improvements in salutogenesis, measured through HRV, in a 43-yearold woman post breast cancer following a course of chiropractic care focused on vertebral subluxation correction. Chiropractic care was provided to a 43-year-old woman following bilateral radical mastectomy and chemotherapy for management of breast cancer. Chiropractic care was focused on the assessment and correction of vertebral subluxation. The parameter numbers used were the standard interbeat interval (Sd IBI) from the heart rate analysis. Chiropractic care was provided over a period of 34 weeks. HRV assessment was performed at the initiation of care and again at weeks 4, 6, 13 and 34. The Sd IBI measurements recorded during the respective assessment visits indicated significant improvement compared to normative data for the same population. A course of chiropractic care focused on the assessment and correction of vertebral subluxation was associated with a salutogenic response in a woman post breast cancer, as measured via HRV.
\end{abstract}

Keywords: Chiropractic; Heart rate variability; Breast cancer

\section{Introduction}

Measured heart rate variability (HRV) has been documented as a reliable assessment tool for predictive longevity in people with cancer. De Couck et al [1] examined the relationship of vagal activity, as shown by HRV, in patients with prostate

\footnotetext{
Manuscript submitted May 10, 2019, accepted June 7, 2019

aPalmer College of Chiropractic, Davenport, IA, USA

bPrivate Practice, Cortland, NY, USA

'Private Practice, Auckland, New Zealand

${ }^{\mathrm{d} S}$ cotland College of Chiropractic Trust, UK

${ }^{e}$ Corresponding Author: Otto J. Janke, Private Practice, Cortland, NY, USA.

Email: drottojanke@gmail.com
}

doi: https://doi.org/10.14740/jmc3306 cancer and found that higher initial HRV was significantly correlated with lower risk of mortality.

Fadul et al [2] described autonomic dysfunction (AD) measured by HRV and its prevalence in advanced cancer. They concluded that $\mathrm{AD}$ is associated with reduced longevity in men with advanced cancer.

A relationship has been established where predictable HRV values, suggesting higher vagal nerve activity, are seen in the survival of patients with cancer [3]. De Couck et al [4] reported that, in people with prostate and non-small cell lung cancer, measuring HRV to determine high vagal activity may be a "routine" measurement that can be considered in estimating prognosis, and that there may be a modulating role of vagal nerve activity in cancer. Women with breast cancer have shown decreased HRV values [5-7]. Neuroimmunomodulation may play a significant factor in the body's ability to recover from cancer and promote longevity [1].

Palma et al [8] reported on 45 women, aged 35 - 70 years, and showed that "breast cancer survivors regardless of their post-operative period exhibited a decrease in overall variability and both sympathetic and parasympathetic activity when compared to women without the disease" [9]. Using HRV could be a valuable tool for "early diagnosis and better prognosis of autonomic dysfunction, and survival in breast cancer patients" [8]. High HRV values relating to autonomic function can help determine longevity, and in the case of women having gone through breast cancer treatments, their HRV values were decreased $[3,5,7,8,10-13]$.

Chiropractic has been shown to have positive impact on HRV studies [14-17]. A core aim of chiropractic care is to optimize the health and wellbeing of individuals through the enhancement of nervous system function brought about by reducing nerve interference caused by vertebral subluxation $[18,19]$. The World Health Organization similarly defines chiropractic as a health care profession concerned with the diagnosis, treatment and prevention of disorders of the neuromusculoskeletal system and the effects of these disorders on general health. There is an emphasis on manual techniques, including joint adjustment and/or manipulation, with a particular focus on subluxations [20]. Vertebral subluxation correction is achieved through chiropractic adjustments that are typically manually performed [20]. Chiropractors commonly use such procedures to influence joint and neurophysiological function [21]. Vertebral subluxations may result in altered autonomic nervous system (ANS) activity [22].

The purpose of this paper was to chronicle the salutogenic 


\section{Autonomic Activity Index: $61.50 \quad$ Autonomic Balance Index: 33.90}

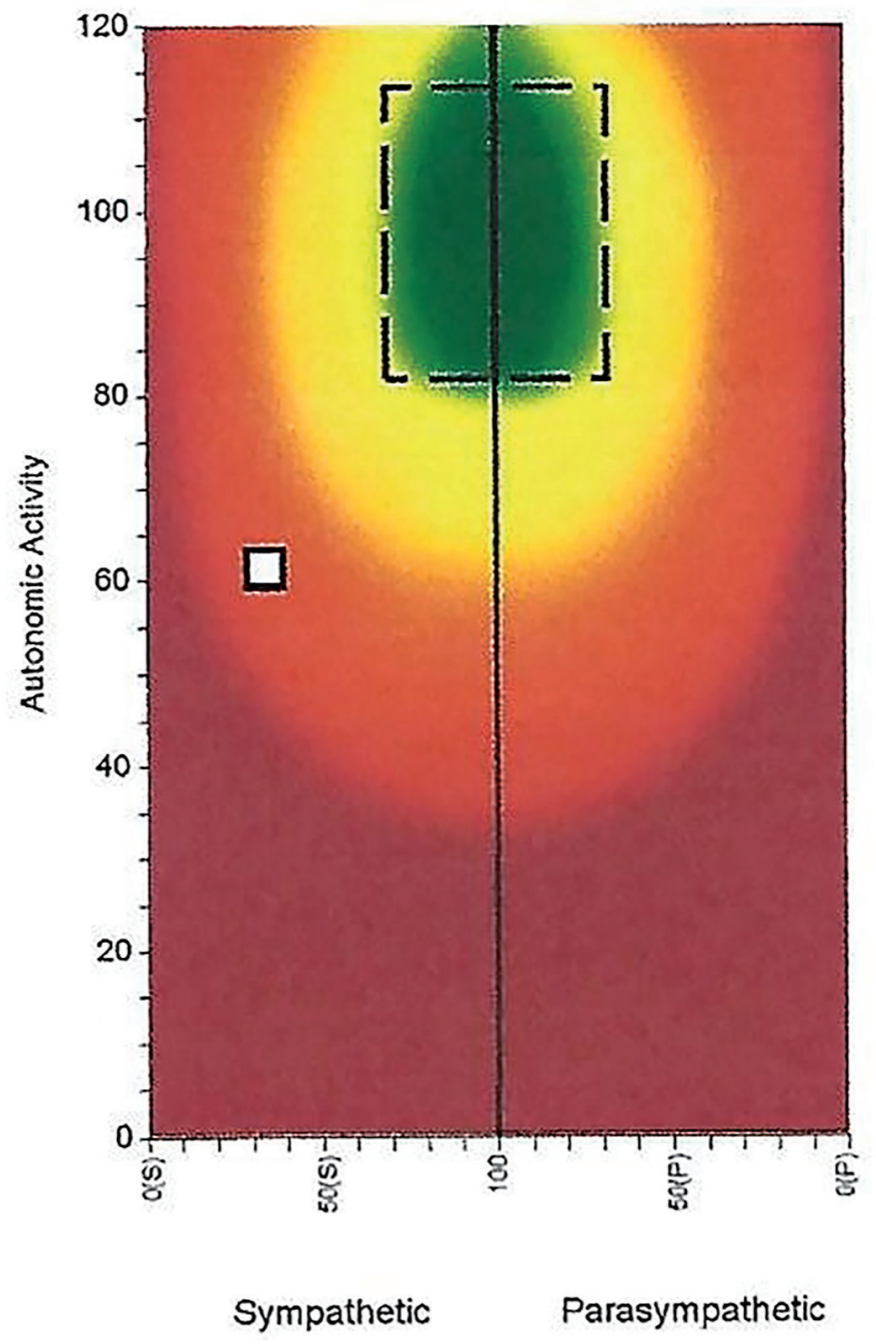

Figure 1. Before starting chiropractic care.

improvements in HRV in a 43-year-old woman, post breast cancer following a course of chiropractic care focused on vertebral subluxation.

\section{Case Report}

\section{History}

A 43-year-old woman presented with a history of double radical mastectomies, including associated lymph nodes removed following diagnosed with type $1 \mathrm{~b}$, stage 3 breast carcinoma. She was diagnosed with a BRCA +1 gene mutation. The mastectomies were performed 12 months prior to presenting for chiropractic care. Following the mastectomies was a 16-week program of chemotherapy, consisting of eight chemotherapy sessions. The chemotherapy formula consisted of adriamycin, cytoxan and taxol. She was primarily sedentary for 8 months during and following the chemotherapy treatments. She reported the classic extremes in fatigue, nausea and indigestion during this time.

The patient's initial presentation for chiropractic care was approximately 8 months after last chemotherapy treatment. At the time of her presentation, she complained of chronic tiredness, overall aches/pain that were non-focal, abnormal tactile sensation in fingers and toes, decreased sensation to touch and temperature in thorax, and "brain fog".

\section{Examination}

A thorough chiropractic examination was performed, including the examination in posture analysis, spinal assessment incorporating neurological and biomechanical assessment indicators of vertebral subluxation [23-25], and HRV as a baseline 


\section{Autonomic Activity Index: $77.00 \quad$ Autonomic Balance Index: 88.20}

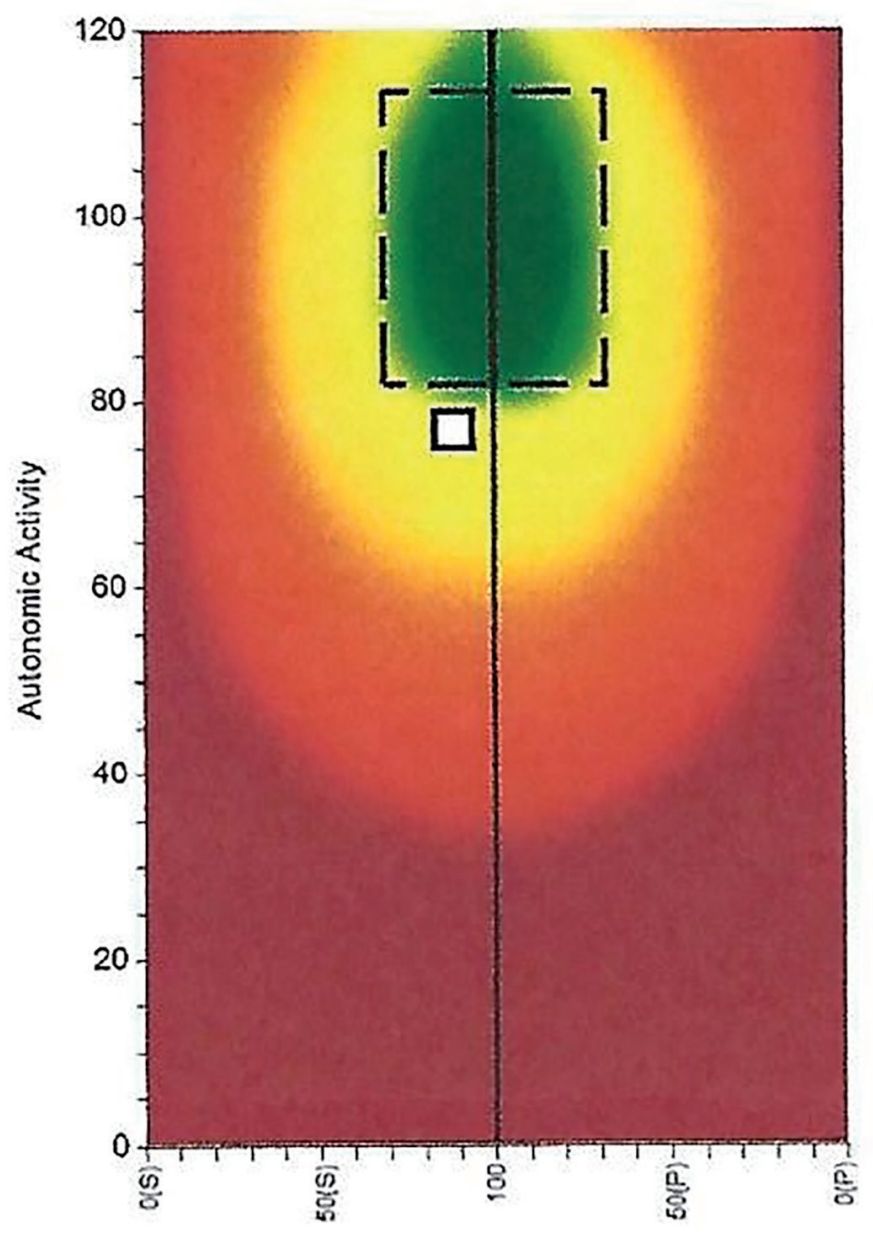

\section{Sympathetic Parasympathetic}

Figure 2. HRV at 4 weeks. HRV: heart rate variability.

and outcome assessment.

\section{HRV}

HRV was collected using the pulse wave profiler as part of the insight subluxation station. The use of HRV allowed examination of the balance between the sympathetic nervous system and the parasympathetic nervous system. "HRV consists of changes in the time intervals between consecutive heartbeats called IBIs. A healthy heart is not a metronome. The oscillations of a healthy heart are complex and constantly changing, which allow the cardiovascular system to rapidly adjust to sudden physical and psychological challenges to homeostasis" [26].

An effective numerical analysis of the HRV graphs was performed using the Sd IBI (ms). The Sd IBI is the standard deviation of the IBI in milliseconds, which measures the spe- cific changes in time between successive heartbeats. The time between beats is called an "R-R interval", or an "IBI" [27]. The outcome numbers when higher are considered better, and lower considered less desirable. HRV assessment revealed a strong imbalance between sympathetic and parasympathetic nervous system (Fig. 1). The number analysis of the Sd IBI of this patient prior to beginning chiropractic care was 45.42.

A simplified explanation of HRV and vagal tone is that a lower measurement is seen as undesirable, while a higher measurement of vagal tone is seen as more desirable. "Research has found that lowered HRV is associated with aging, and increased incidence of sudden death" [14].

\section{Chiropractic management and outcomes}

The patient followed a care plan of 44 visits over 8 months. 


\section{Autonomic Activity Index: $85.40 \quad$ Autonomic Balance Index: 95.30}

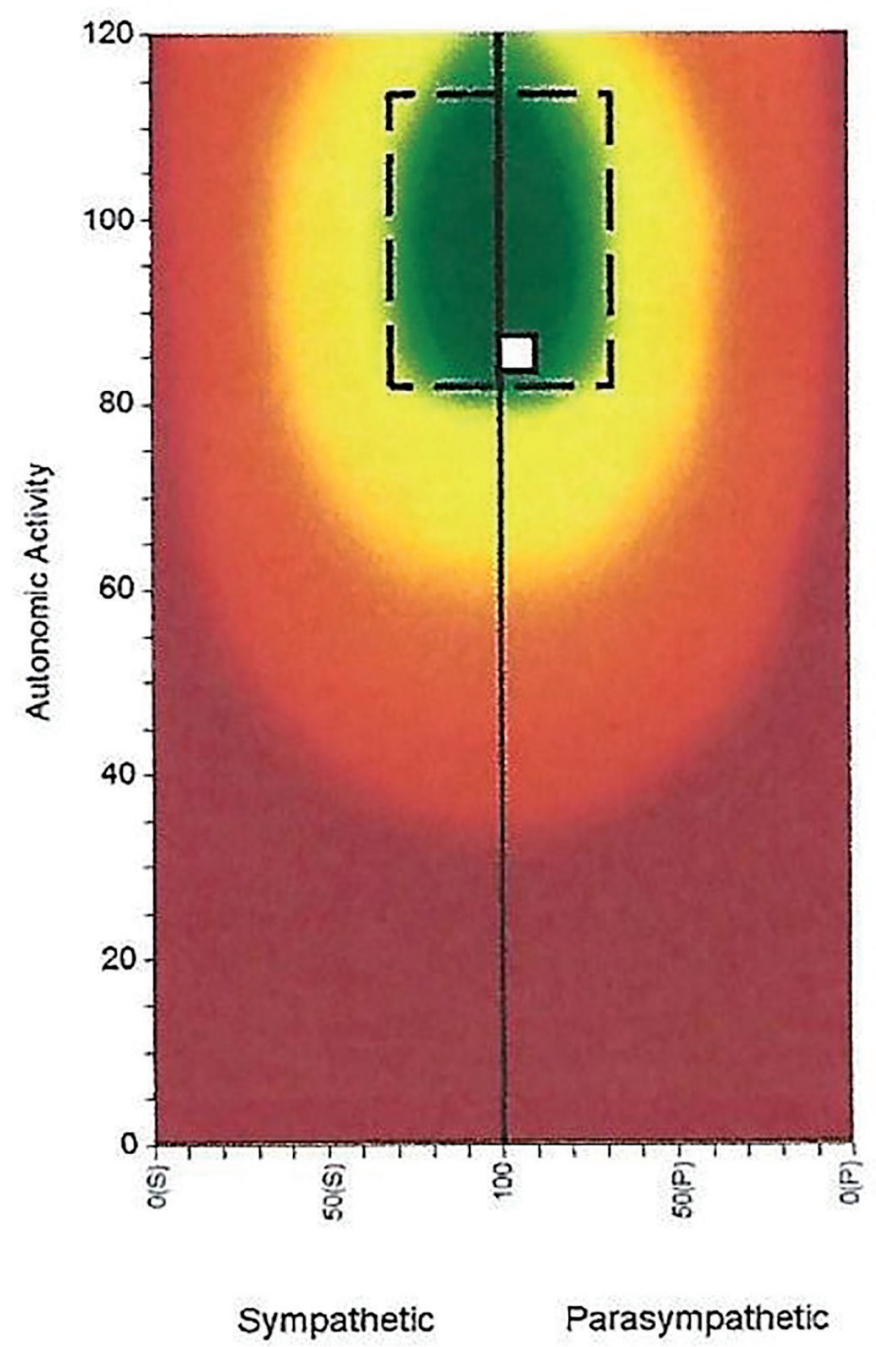

Figure 3. HRV at 6 weeks. HRV: heart rate variability.

To determine the site of vertebral subluxation, the Thompson protocol was used [28]. Thompson technique is a full spine adjusting technique that focuses on the cervical spine and pelvis, and emphasizes high-velocity low-amplitude, and low-force procedures using a weighted drop table. The specific protocol allows the chiropractor to know "where to begin and what specific area to adjust" [28]. The technique had to be modified in adaptation of the patient's physical state.

HRV was the primary examination tool used to objectively evaluate vagal tone and progress of the patient. HRV assessment was performed on the patient four times after the initiation of chiropractic care at 4 weeks, 6 weeks, 3 months and 6 months and showed sustained improvement. The number analysis of the Sd IBI of this patient improved from an initial calculation of 45.42 , and progressed to 128.58 . The higher the number recorded, the better the vagal tone. The number analysis of the Sd IBIs at each assessment interval was 74.05 (4 weeks), 124.42 (6 weeks), 114.43 (3 months) and 128.58 (6 months) (Figs. 2-5).

\section{Discussion}

A 43-year-old woman who followed a protocol for breast cancer treatment presented for chiropractic care with a wide range of neurological, postural and orthopedic signs and symptoms. A course of chiropractic care was associated with sustained improvement in HRV assessments.

Studies show that HRV of women post breast cancer is dramatically low, even with following appropriate protocols $[5,13,26]$. It is known that low vagal activity is a predictor of onset and progression of diseases such as cancer, and that this is reason to activate the vagus nerve by therapeutic intervention [29]. Chiropractic care has been shown to improve HRV, and therefore should be considered as an intervention [14-17, 30-32]. Studies show that "biomechanical changes caused by 


\section{Autonomic Activity Index: $83.50 \quad$ Autonomic Balance Index: 93.50}

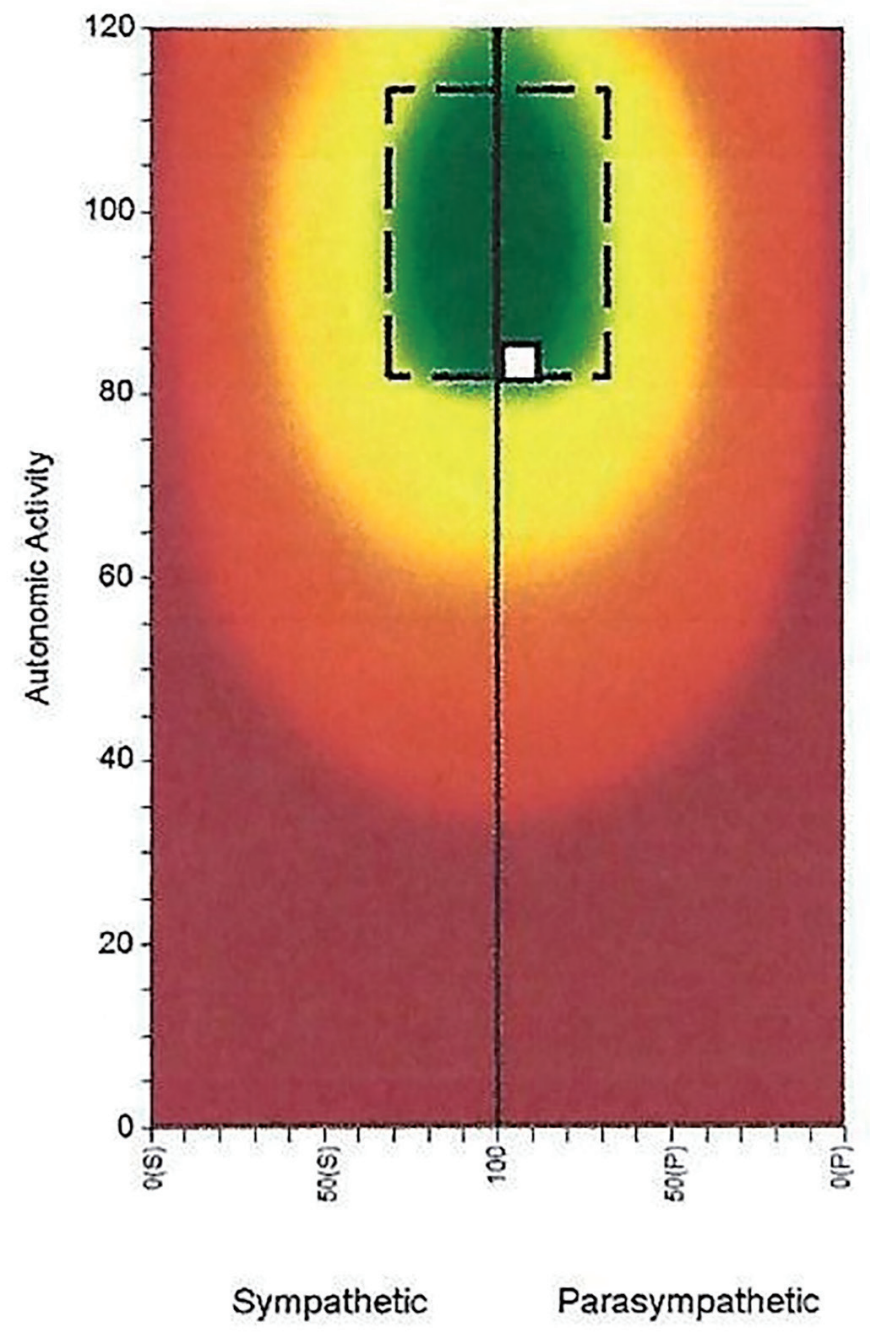

Figure 4. HRV at 3 months. HRV: heart rate variability.

spinal manipulation are thought to have physiological consequences by means of their effects on the inflow of sensory information to the central nervous system" [33]. This effect on the central nervous system has the opportunity for systemic improvement. "Spinal manipulation is a therapeutic technique used by manual therapists which may have widespread neurophysiological effects" [34].

The use of complementary and alternative medicine (CAM), which includes chiropractic care, is increasing with cancer patients [35]. The use of chiropractic in the care of cancer patients is, however, scant with one study describing the use of CAM at a cancer center [35]. The focus of the research found in PubMed relating to "chiropractic" and "cancer" related to musculoskeletal pain associated with the cancer [36-38]. No studies were found relating to "chiropractic" and "cancer" and "HRV" suggesting the novel approach investigated in the current study.

Winn et al [30] showed ANS responses via HRV when manipulating either the upper cervical spine ( $\mathrm{C} 1$ and $\mathrm{C} 2)$, or lower (C6 and C7) segments. Driscoll et al [31] showed the chiropractic manipulative therapy (CMT) improved autonomic function assessed using electrocardiogram and arterial tonometry. Roy et al [32] used HRV to evaluate the parasympathetic nervous system and found change after a single session of chiropractic care.

A number of recent studies have documented an association between chiropractic care and positive changes in HRV values. Knowles et al [16] reported a retrospective study of 46 patients evaluated with HRV while undergoing a program of care using network spinal analysis, showing it "may be an effective method for people to improve their ANS function through adjustments of vertebral subluxation, reorganizing spinal tension patterns and decreasing physiological stress" [16]. Haas and Russell [15] reported sustained improvement in HRV was seen up to 2 years when patients underwent chiropractic care. Zhang et al [14] concluded in their study "... 


\section{Autonomic Activity Index: $89.10 \quad$ Autonomic Balance index: 76.40}

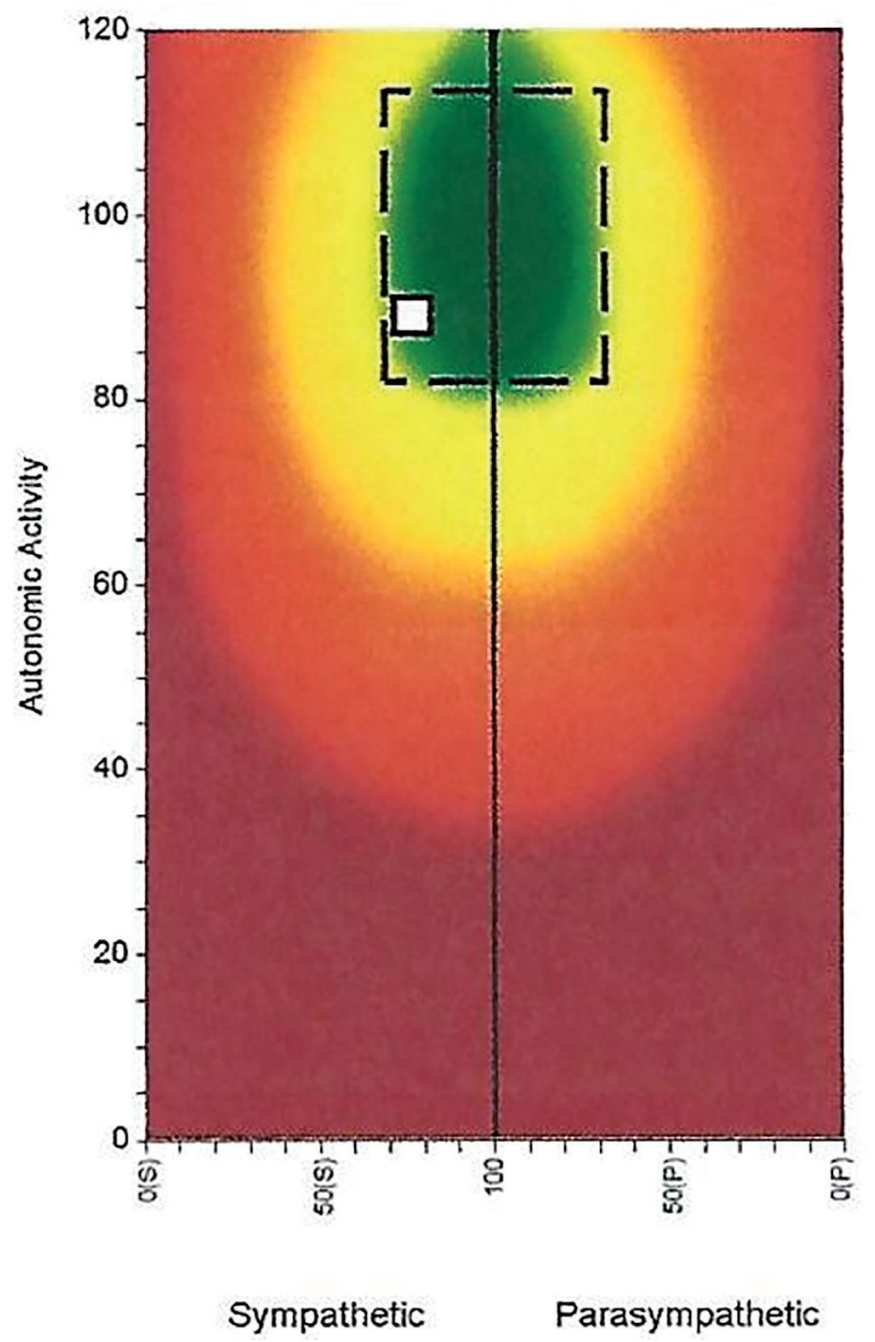

Figure 5. HRV at 6 months. HRV: heart rate variability.

HRV and visual analog scale (VAS) changed in patients as a result of chiropractic care". Hart [17] concluded HRV measures demonstrate chiropractic improved neurological function and adaptability. The positive changes documented in the current case reflect those previously reported [14-17]. To our knowledge, no prior studies demonstrate improved HRV in post breast cancer patients receiving chiropractic care, highlighting that further research is needed to investigate the role that chiropractors may play in assisting similar patient populations so as to inform clinical practice and future higher-level research designs.

\section{Limitations}

This is a single case report. Due to the inherent limitations, being an isolated case not controlled for external factors and natural progression, the findings cannot be generalized or cau- sation of vertebral subluxation implied. To further gauge the effectiveness of chiropractic care for a homogeneous population, further studies are needed.

\section{Conclusion}

A course of chiropractic care focused on the assessment and correction of vertebral subluxation was associated with a salutogenic response in a woman post breast cancer, as measured via HRV.

\section{Acknowledgments}

This study was presented as abstract at the International Conference on Euro Oncology, Breast Cancer \& Biomarkers in Amsterdam, Netherlands (October 2018). Authors thank Da- 
vid Fletcher, DC President and CEO of Chiropractic Leadership Alliance for his guidance, expertise, and insight.

\section{Financial Disclosure}

There has been no funding for this study.

\section{Conflict of Interest}

Authors declare that they have no conflict of interest to declare.

\section{Informed Consent}

Informed consent was obtained from the individual participant in this study.

\section{Author Contributions}

Both authors made substantial contributions to acquisition of data, or analysis and interpretation of data; had substantial involvement in drafting the manuscript and revising it critically for important intellectual content. Both authors read and approved the final manuscript.

\section{References}

1. De Couck M, Marechal R, Moorthamers S, Van Laethem JL, Gidron Y. Vagal nerve activity predicts overall survival in metastatic pancreatic cancer, mediated by inflammation. Cancer Epidemiol. 2016;40:47-51.

2. Fadul N, Strasser F, Palmer JL, Yusuf SW, Guo Y, Li Z, Allo J, et al. The association between autonomic dysfunction and survival in male patients with advanced cancer: a preliminary report. J Pain Symptom Manage. 2010;39(2):283-290.

3. Zhou X, Ma Z, Zhang L, Zhou S, Wang J, Wang B, Fu W. Heart rate variability in the prediction of survival in patients with cancer: A systematic review and meta-analysis. J Psychosom Res. 2016;89:20-25.

4. De Couck M, van Brummelen D, Schallier D, De Greve J, Gidron Y. The relationship between vagal nerve activity and clinical outcomes in prostate and non-small cell lung cancer patients. Oncol Rep. 2013;30(5):2435-2441.

5. Caro-Moran E, Fernandez-Lao C, Galiano-Castillo N, Cantarero-Villanueva I, Arroyo-Morales M, Diaz-Rodriguez L. Heart rate variability in breast cancer survivors after the first year of treatments: a case-controlled study. Biol Res Nurs. 2016;18(1):43-49.

6. Vigo C, Gatzemeier W, Sala R, Malacarne M, Santoro A, Pagani M, Lucini D. Evidence of altered autonomic cardiac regulation in breast cancer survivors. J Cancer Surviv. 2015;9(4):699-706.

7. De Couck M, Gidron Y. Norms of vagal nerve activity, in- dexed by Heart Rate Variability, in cancer patients. Cancer Epidemiol. 2013;37(5):737-741.

8. Palma MR, Vanderlei LC, Ribeiro FE, Mantovani AM, Christofaro DG, Fregonesi CE. The relationship between post-operative time and cardiac autonomic modulation in breast cancer survivors. Int J Cardiol. 2016;224:360-365.

9. Arab C, Dias DP, Barbosa RT, Carvalho TD, Valenti VE, Crocetta TB, Ferreira M, et al. Heart rate variability measure in breast cancer patients and survivors: A systematic review. Psychoneuroendocrinology. 2016;68:57-68.

10. Gidron Y, De Couck M, De Greve J. If you have an active vagus nerve, cancer stage may no longer be important. J Biol Regul Homeost Agents. 2014;28(2):195-201.

11. Yamazoe T, Okanishi T, Yamamoto A, Yamada T, Nishimura M, Fujimoto A, Enoki H, et al. New-onset refractory status epilepticus treated with vagus nerve stimulation: A case report. Seizure. 2017;47:1-4.

12. Giese-Davis J, Wilhelm FH, Tamagawa R, Palesh O, Neri E, et al. Higher Vagal activity as related to survival in patients with advanced breast cancer: an analysis of autonomic dysregulation. Psychosom Med. 2015;77(4):346355.

13. Crosswell AD, Lockwood KG, Ganz PA, Bower JE. Low heart rate variability and cancer-related fatigue in breast cancer survivors. Psychoneuroendocrinology. 2014;45:58-66.

14. Zhang J, Dean D, Nosco D, Strathopulos D, Floros M. Effect of chiropractic care on heart rate variability and pain in a multisite clinical study. J Manipulative Physiol Ther. 2006;29(4):267-274.

15. Haas A, Russell D. Improved nerve function, adaptability \& resilience following chiropractic care. Chiro J Austr. 2017;45(4): 338-358.

16. Knowles D, Knowles R, Kotur D. Improvement in heart rate variability in 46 patients undergoing chiropractic with network spinal analysis: a retrospective analysis of outcomes. A Vertebral Subluxation Res. 2017;1:232-237.

17. Hart J. Heart rate variability following spinal adjustment: a practice-based study. The Internet Journal of Neurology. 2019;21(1):1-8.

18. Haavik H, Holt K, Murphy B. Exploring the neuromodulatory effects of vertebral subluxation and chiropractic care. Chiropr J Aust. 2010;40(1):37-44.

19. Russell D. The assessment and correction of vertebral subluxation is central to chiropractic Practice: Is there a gap in the clinical evidence? J Contemporary Chiropr. 2019;2(1):4-17.

20. World Federation of Chiropractic. Definitions of Chiropractic. 2015. Available from: https://www.wfc.org/website/index.php?option $=$ com_content $\& v i e w=$ article $\& i d=9$ 0\&Itemid=110 [Accessed $1 \overline{8}$ November 2018].

21. Shafiq H, McGregor C, Murphy B. The impact of cervical manipulation on heart rate variability. Conf Proc IEEE Eng Med Biol Soc. 2014;2014:3406-3409.

22. Kent C. Heart rate variability to assess the changes in autonomic nervous system function associated with vertebral subluxation. Review Article. Research \& Reviews: Neuroscience. 2017;1(3):14-21.

23. Triano J, Budgell B, Bagnulo A, Roffey B, Bergmann T, 
et al. Review of methods used by chiropractors to determine the site for applying manipulation. Chiropr Man Ther. 2013:21(36):1-29.

24. Holt K, Russell D, Cooperstein R, Young M, Sherson M, et al. Interexaminer reliability of a multidimensional battery of tests used to assess for vertebral subluxation. Chiropr J Aust. 2018;46(1):100-117.

25. Owens E. Chiropractic subluxation assessment: What the research tells us. J Can Chiro Assoc. 2002;46(4):215-220.

26. Giese-Davis J, Wilhelm FH, Tamagawa R, Palesh O, Neri E, Taylor CB, Kraemer HC, et al. Higher vagal activity as related to survival in patients with advanced breast cancer: an analysis of autonomic dysregulation. Psychosom Med. 2015;77(4):346-355.

27. Shaffer F, Ginsberg JP. An overview of heart rate variability metrics and norms. Front Public Health. 2017;5:258.

28. Cooperstein R. Technique system overview: Thompson Technique. Chiropractic Technique. 1995;7(2):60-63.

29. De Couck M, Cserjesi R, Caers R, Zijlstra WP, Widjaja D, Wolf N, Luminet O, et al. Effects of short and prolonged transcutaneous vagus nerve stimulation on heart rate variability in healthy subjects. Auton Neurosci. 2017;203:8896.

30. Win NN, Jorgensen AM, Chen YS, Haneline MT. Effects of upper and lower cervical spinal manipulative therapy on blood pressure and heart rate variability in volunteers and patients with neck pain: a randomized controlled, cross-over, preliminary study. J Chiropr Med.
2015;14(1):1-9.

31. Driscoll MD, Hall MJ. Effects of spinal manipulative therapy on autonomic activity and the cardiovascular system: a case study using the electrocardiogram and arterial tonometry. J Manipulative Physiol Ther. 2000;23(8):545550.

32. Roy RA, Boucher JP, Comtois AS. Heart rate variability modulation after manipulation in pain-free patients vs patients in pain. J Manipulative Physiol Ther. 2009;32(4):277-286.

33. Pickar JG. Neurophysiological effects of spinal manipulation. Spine J. 2002;2(5):357-371.

34. Kovanur Sampath K, Mani R, Cotter JD, Tumilty S. Measureable changes in the neuro-endocrinal mechanism following spinal manipulation. Med Hypotheses. 2015;85(6):819-824.

35. Subramani R, Lakshmanaswamy R. Complementary and alternative medicine and breast cancer. Prog Mol Biol Transl Sci. 2017;151:231-274.

36. Evans RC, Rosner AL. Alternatives in cancer pain treatment: the application of chiropractic care. Semin Oncol Nurs. 2005;21(3):184-189.

37. Luo Q, Asher GN. Complementary and alternative medicine use at a comprehensive cancer center. Integr Cancer Ther. 2017;16(1):104-109.

38. Kanga I, Steiman I. Chiropractic management of a patient with breast cancer metastases to the brain and spine: a case report. J Can Chiropr Assoc. 2015;59(3):269-278. 